\title{
Prevalence of Inflammatory Rheumatic Diseases in a Rheumatologic outpatient clinic: analysis of 12626 cases
}

\author{
Mohammadhassan Jokar ${ }^{1 *}$, Mina Jokar ${ }^{2}$ \\ ${ }^{l}$ Faculty of Medicine, Mashhad University of Medical Sciences, Mashhad, Iran \\ ${ }^{2}$ Faculty of Medicine, Islamic Azad University, Mashhad Branch, Iran
}

\begin{abstract}
Inflammatory rheumatic diseases are a heterogeneous class of often chronic autoimmune disorders. They are among the most common chronic diseases. They cause major health problems in the general population. This study assessed the distribution of inflammatory systemic rheumatic diseases in a rheumatologic outpatient clinic. The medical records of patients diagnosed with any type of inflammatory rheumatic disease between January 1, 2006 and December 31, 2016 in a non-hospital-based rheumatologic outpatient practice in Mashhad, Iran were retrospectively studied. Diagnoses were made using the agreed-upon classification criteria. Data regarding each patient's diagnosis, age at onset of disease, and gender was extracted from their files. The total number of patients was 12,626. The most common diseases were rheumatoid arthritis (47.30\%), spondyloarthropathies $(17.23 \%)$, systemic lupus erythematosus $(8.10 \%)$, gout $(7.84 \%)$, and vasculitis $(6.84 \%)$. Patients were aged from 1 to 93 years, with a mean age of $41.17 \pm 39.70$ years. Most patients were in the third, fourth, and fifth decade of life. Sixty-four percent of all patients were female. The overall sex ratio (women to men) was 1.8:1. The proportion of women was $95 \%$ in Takayasu's arteritis, $92 \%$ in systemic lupus erythematosus, $87 \%$ in Sjögren's syndrome, $78 \%$ in rheumatoid arthritis, and $24 \%$ in ankylosing spondylitis. The age at onset of inflammatory rheumatic diseases in Mashhad, Iran is lower than that in some other regions. The frequency of Behcet's disease, systemic lupus erythematosus, and systemic sclerosis was greater in this study than in most other studies, but gout, polymyalgia rheumatica, and psoriatic arthritis were less frequent in the current study.
\end{abstract}

Keywords: arthritis, epidemiology, inflammatory, rheumatic diseases, rheumatology.

\section{Introduction}

Rheumatic disorders are among the most prevalent chronic diseases of the musculoskeletal system and connective tissue, and they can affect a wide range of age groups. Encompassing a large number of arthritis and autoimmune diseases, they can affect the bones, joints, and other components of the musculoskeletal system, causing morbidity or disability with resultant healthcare utilization [1]. Rheumatic disorders are mainly responsible for an inability to work and early retirement, a fact which highlights their enormous social and economic impact [2]. The economic burden of rheumatic diseases is often more substantial than other chronic conditions, including cardiovascular diseases and cancer [3]. Unfortunately, despite the growing disease burden associated with rheumatic diseases, inadequate attention has been paid to them and to arthritis in the scientific literature [4].

There are more than 150 classified rheumatic disease conditions with specific pathogenesis, clinical picture, treatment, and prognosis. For successful treatment, identifying each condition and its variations is essential [1]. Rheumatic diseases can be divided into two major groups: inflammatory rheumatic diseases (IRDs) and non-inflammatory rheumatic diseases. As the most common rheumatic diseases, non-inflammatory rheumatic diseases are highly age-dependent and usually have a better prognosis [5]. About 5\% of the population, however, suffer from a chronic inflammatory rheumatic disease [6]. Statistics show that, in America, a higher number of disabilities are caused by inflammatory rheumatic diseases with arthritis than by heart disease, cancer, or diabetes [7].

As a heterogeneous group of often chronic immunemediated disorders, inflammatory rheumatic diseases cause inflammatory reactions in various body tissues. The primary target is the musculoskeletal system; these disorders cause joint pain (arthralgia) and restricted mobility, leading to irreversible damage and disability. Some internal organs, including the heart and kidneys,

\footnotetext{
* Corresponding Author: Mohammadhassan Jokar, Email: jokarmh@mums.ac.ir, Tel: +98 09155178351, Fax: +98 5138413006

Received: 03 July 2017; Accepted: 04 September 2017
} 
can also be affected [8, 9]. There are over 30 autoimmune rheumatic diseases; some of the most common ones are rheumatoid arthritis, lupus, scleroderma, juvenile idiopathic arthritis, Sjögren's syndrome, spondyloarthropathies, polymyalgia rheumatica, and systemic vasculitis [10].

The epidemiological and demographic features of vasculitis [11], giant cell arteritis [12], Takayasu's arteritis [13], and sarcoidosis [14] in northeastern Iran have previously been reported. This study aimed to analyze the inflammatory rheumatic disease profile of patients visiting an outpatient rheumatology practice in Mashhad, Iran and compare the prevalence and distribution of different inflammatory rheumatic diseases.

\section{Materials and Methods}

The medical records of patients diagnosed with any type of inflammatory rheumatic disease between January 1, 2006 and December 31, 2016 in a non-hospital-based rheumatologic outpatient practice in Mashhad, Iran were retrospectively studied.

The following disorders were defined as inflammatory rheumatic diseases: rheumatoid arthritis (RA), systemic lupus erythematosus (SLE), systemic sclerosis (SSc), spondyloarthropathies, Sjogren's syndrome (SS), dermatomyositis/ polymyositis, relapsing polychondritis, sarcoidosis, vasculitides, adult onset Still's disease, juvenile idiopathic arthritis (JIA), antiphospholipid antibody syndrome (APS), crystalinduced arthritis, familial Mediterranean fever (FMF), acute rheumatic fever, polymyalgia rheumatica (PMR), SAPHO (Synovitis-acne-pustulosis-hyperostosisosteitis), RS3PE (remitting seronegative symmetrical synovitis with pitting edema), mixed connective tissue disease (MCTD), and palindromic rheumatism.

The following conditions were defined as noninflammatory conditions and were excluded from this study: osteoarthritis, osteoporosis, noninflammatory back pain, soft tissue rheumatism, complex regional pain syndrome, fibromyalgia, malignancy and hypertrophic osteoarthropathy. Patients with infectious arthritis were also excluded.

The agreed classification criteria were used to make the diagnosis (Table 1). Data regarding diagnosis, age at disease onset, and gender was extracted from the patients' files.

\section{Statistical analysis}

SPSS software (Statistical Package for the Social Sciences) 20 was used for data entry and analysis. Continuous data was shown as mean and standard deviation (mean $\pm \mathrm{SD}$ ), and categorical variables were shown as percentages.

\section{Results}

The total number of patients with inflammatory rheumatic disease was 12,626. Table 2 presents the distribution of patients separately grouped based on their diagnoses. The most common diseases were RA (47.30\%), spondyloarthropathies (including ankylosing spondylitis, psoriatic arthritis, reactive arthritis and enteropathic arthritis) (17.23\%), SLE (8.10\%), gout $(7.84 \%)$, and vasculitis $(6.83 \%)$.

Patients' ages ranged from 1 to 93 years, with a mean of $41.17 \pm 39.70$ years. Most patients were in the third, fourth, or fifth decade of life. Figure 1 shows the distribution of age at disease onset in the studied patients.

Sixty four percent of all patients were female. The overall sex ratio (women to men) was 1.8:1. The proportion of women was $95 \%$ in Takayasu's arteritis, $92 \%$ in SLE, $87 \%$ in Sjögren's syndrome, $78 \%$ in RA, and $24 \%$ in AS. The most common age of onset for AS, reactive arthritis (ReA), Takayasu's arteritis, Still's disease, Behcet's disease, and SLE was in the twenties, whereas the most common age of onset for Granulomatosis with polyangiitis (GPA), sarcoidosis, psoriatic arthritis (PsA), APS, inflammatory bowel disease (IBD), palindromic rheumatism, and systemic sclerosis was in the thirties. The most common age of onset for RA was in the forties. The total number of patients with JIA was $359(2.8 \%)$.

\section{Discussion}

The exact etiology and pathogenesis of inflammatory rheumatic diseases remain unclear today. However, among a host of factors, a variable combination of individual genetic predisposition, environmental factors, and dysregulated immune responses have been singled out as the underlying causes of these autoimmune diseases [15]. The role of genetic predisposition, in particular the influence of distinct HLA haplotypes, has been highlighted in most of these diseases. Moreover, environmental factors including nutrition, infection, and exposure to sunlight have been pinpointed as being responsible for the development of the disease [16].

A number of reports on the epidemiology and prevalence of rheumatic diseases seen in rheumatology practices have been published [17-20]. The inflammatory rheumatic patients in this study were compared with other populations in Germany [17], the Netherlands [18], Belgium [19], and Nigeria [20] (Table 3). 
Table 1. The used classification criteria for inflammatory rheumatic diseases

\begin{tabular}{|c|c|}
\hline Disease & Classification criteria \\
\hline Acute rheumatic fever & $\begin{array}{l}2004 \text { World Health Organization Criteria for the Diagnosis of Rheumatic Fever and } \\
\text { Rheumatic Heart Disease }\end{array}$ \\
\hline Adult onset Still's disease & Yamaguchi criteria \\
\hline Antiphospholipid antibody syndrome & Revised Sapporo Classification Criteria for Anti-phospholipid Syndrome \\
\hline Ankylosing Spondylitis & Modified New York, 1984 \\
\hline Behcet's disease & Revised International Criteria for Behçet’s Disease \\
\hline Cryoglobulinemic vasculitis & Preliminary classification criteria for the cryoglobulinaemic vasculitis \\
\hline CPPD & McCarty and colleagues \\
\hline Dermatomyostis/ Polymyositis & Bohan and Peter Criteria for Polymyositis and Dermatomyositis \\
\hline $\begin{array}{l}\text { Eosinophilic granulomatosis with } \\
\text { polyangiitis }\end{array}$ & ACR 1990 criteria for Churg-Strauss syndrome \\
\hline Familial Mediterranean Fever & Tel Hashomer Medical Center \\
\hline Giant cell arteritis & American College of Rheumatology Classification Criteria for Giant Cell Arteritis \\
\hline Gout & ACR/EULAR \\
\hline Granulomatosis with polyangiitis & ACR criteria \\
\hline $\begin{array}{l}\text { Hypocomplementemic urticarial } \\
\text { vasculitis }\end{array}$ & 1982 Schwartz et al. \\
\hline Inclusion body Myositis & The proposed European Neuromuscular Centre (ENMC) 2011 \\
\hline IgA Vasculitis & $\begin{array}{l}\text { American College of Rheumatology } 1990 \text { Criteria for the Classification of Henoch- } \\
\text { Schönlein Purpura }\end{array}$ \\
\hline Juvenile Idiopathic Arthritis & IL AR Classification Criteria for Juvenile Idiopathic Arthritis \\
\hline Microscopic polyangiitis & ACR 1990 \\
\hline Mixed Connective Tissue Disease & Alarcón-Segovia Criteria \\
\hline Palindromic rheumatism & Pasero and Barbieri \\
\hline Polyarteritis nodosa & American College of Rheumatology Criteria for Classification of Polyarteritis Nodosa \\
\hline Polymyalgia Rheumatica & ACR/EULAR 2012 provisional classification criteria for Polymyalgia rheumatica \\
\hline Polychondritis & Modified (Damiani) criteria \\
\hline Psoriatic Arthritis & CASPAR Classification Criteria for Psoriatic Arthritis \\
\hline Reactive arthritis & French Society of Rheumatology (FSR) \\
\hline Rheumatoid arthritis & ACR/EULAR 2010 \\
\hline RS3PE & Olive criteria \\
\hline SAPHO & Benhamou criteria \\
\hline Sarcoidosis & Visser's Criteria for Sarcoidosis in Patients with Arthritis and Bihilar Lymphadenopathy \\
\hline Sjogren's syndrome & Revised International Classification Criteria for Sjogren’s Syndrome \\
\hline Systemic lupus erythematosus & SLICC criteria for the classification of systemic lupus erythematosus \\
\hline Systemic sclerosis & $\begin{array}{l}\text { American College of Rheumatology/European League against Rheumatism Classification } \\
\text { Criteria for the Classification of Systemic Sclerosis }\end{array}$ \\
\hline Takayasu' arteritis & American College of Rheumatology Classification Criteria for Takayasu's Arteritis \\
\hline $\begin{array}{l}\text { Undifferentiated seronegative } \\
\text { spondyloarthropathy }\end{array}$ & ESSG criteria \\
\hline Vasculitis & Revised International, Chapel Hill Consensus Conference Nomenclature of Vasculitides \\
\hline
\end{tabular}


Inflammatory rheumatic diseases

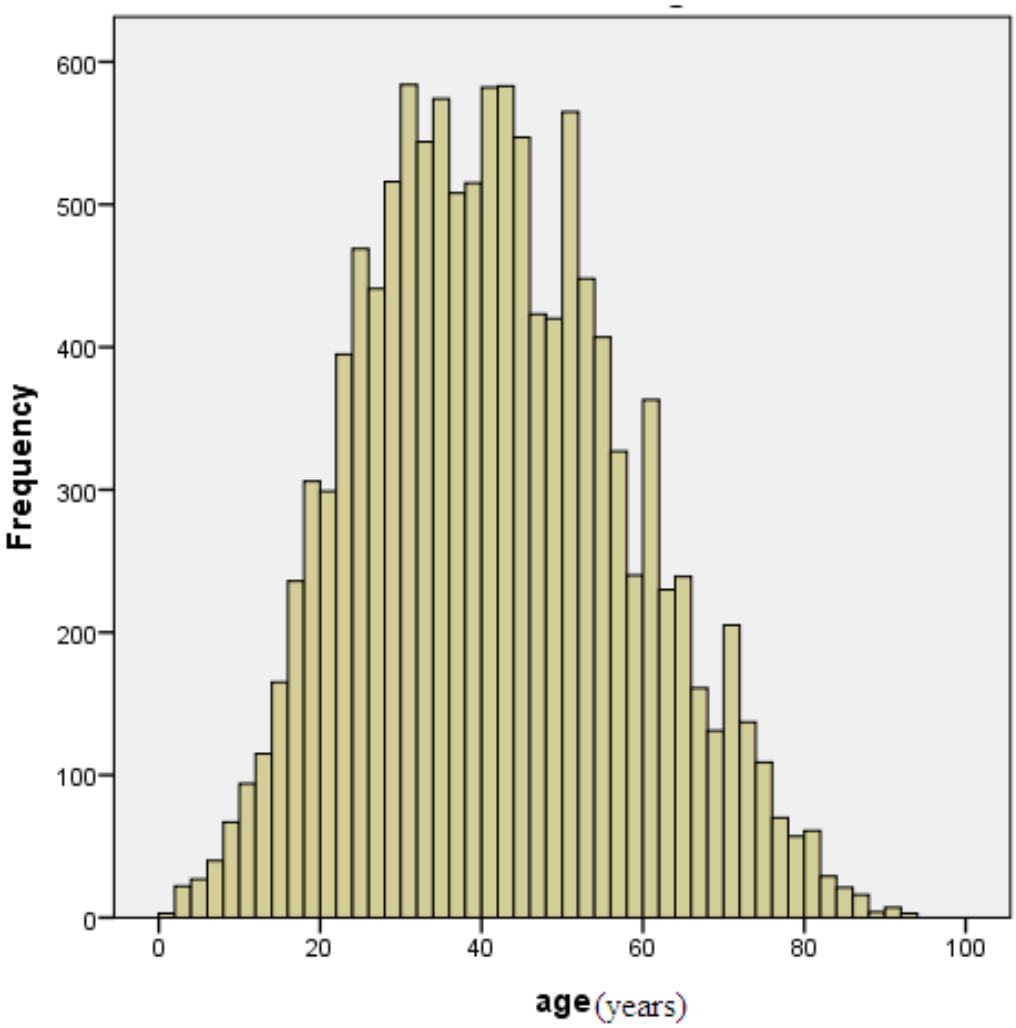

Fig. 1. Age distribution of the patients

Table 2. Distribution of patients and their diagnoses, female \% and ages

\begin{tabular}{|c|c|c|c|c|c|c|c|}
\hline \multirow{2}{*}{\multicolumn{3}{|c|}{ Disaese }} & \multirow{2}{*}{ Frequency no. (\%) } & \multirow{2}{*}{ Female (\%) } & \multicolumn{3}{|c|}{ Age (years) } \\
\hline & & & & & Minimum & Maximum & Mean \\
\hline RA & & & $5973(47.30)$ & 78.5 & 16 & 90 & 46.69 \\
\hline SLE & & & $1023(8.10)$ & 92.0 & 3 & 74 & 31.74 \\
\hline \multirow{6}{*}{ SPA } & Total & & $2176(17.23)$ & 42.0 & 3 & 76 & 33.44 \\
\hline & AS & & $665(5.26)$ & 24.2 & 9 & 75 & 34.12 \\
\hline & $\operatorname{ReA}$ & & $113(0.89)$ & 32.7 & 8 & 71 & 28.45 \\
\hline & PsA & & 373 (2.95) & 59.0 & 3 & 76 & 38.47 \\
\hline & Enterpathic & & $104(0.82)$ & 57.7 & 17 & 74 & 37.06 \\
\hline & USPA & & 921 (7.29) & 1.37 & 5 & 72 & 31.12 \\
\hline \multirow{4}{*}{ Inflammatory myopathies } & Total & & 167 (1.32) & 60.2 & 3 & 74 & 36.29 \\
\hline & $\mathrm{DM}$ & & $67(0.53)$ & 48.1 & 3 & 68 & 38.43 \\
\hline & PM & & 99 (o.78) & 78.1 & 8 & 70 & 34.75 \\
\hline & IBM & & $1(0.00007)$ & 100 & 74 & 74 & 74.00 \\
\hline SSc & & & $299(2.36)$ & 79.9 & 4 & 85 & 40.25 \\
\hline SS & & & $111(0.87)$ & 87.4 & 16 & 75 & 46.44 \\
\hline PMR & & & $52(0.41)$ & 51.9 & 40 & 84 & 66.42 \\
\hline Vasculitidies & Total & & $864(6.84)$ & 51.5 & 5 & 86 & 34.54 \\
\hline \multirow{3}{*}{ LVV } & Total & & $95(0.75)$ & 72.9 & 17 & 83 & 45.49 \\
\hline & TAK & & $50(0.38)$ & 95.7 & 17 & 48 & 28.33 \\
\hline & GCA & & $45(0.35)$ & 46.2 & 44 & 83 & 64.19 \\
\hline \multirow{3}{*}{ MVV } & Total & & $23(0.18)$ & 53.4 & 11 & 82 & 41.00 \\
\hline & PAN & & $23(0.18)$ & 50.0 & 11 & 82 & 41.00 \\
\hline & $\mathrm{KD}$ & & $0(0)$ & - & - & - & - \\
\hline \multirow{4}{*}{ SVV } & Total & & $138(1.09)$ & 48.5 & 5 & 66 & 34.23 \\
\hline & & Total & $83(0.65)$ & 51.5 & 11 & 66 & 35.90 \\
\hline & AAV & GPA & $49(0.38)$ & 50.0 & 11 & 61 & 33.00 \\
\hline & & EGPA & $23(0.18)$ & 33.3 & 23 & 66 & 51.07 \\
\hline
\end{tabular}




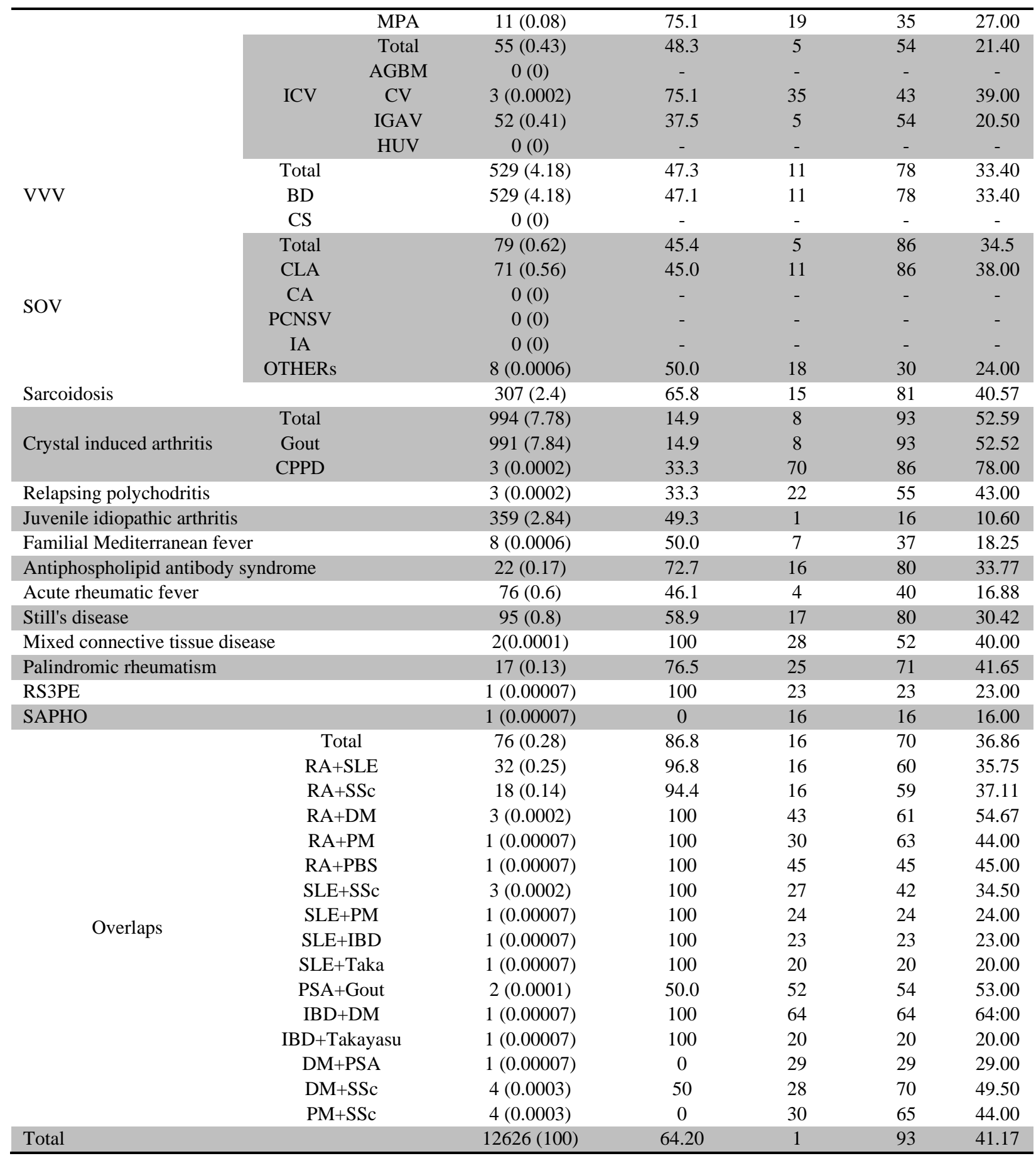

RA: Rheumatoid Arthritis; SLE: Systemic Lupus Erythamtosus; SPA: spondyloarthropathies; AS: Ankylosing Spondylitis; ReA: Reactive Arthritis, PsA: Psoriatic Arthritis; USPA: undifferentiated spondyloarthropathies; DM: dermatomyositis; PM: polymyositis; IBM: Inclusion Body Myositis; SS: Sjogren's Syndrome; SSc: Systemic Sclerosis; PMR: polymyalgia rheumatica; LVV: Large Vessel Vasculitis; TAK: Takayasu arteritis; GCA: Giant Cell Arteritis; MVV: medium vessel vasculitis; PAN: polyarteritis nodosa; KD: Kawasaki Disease; SVV: Small Vessel Vasculitis; AAV: antineutrophil cytoplasmic antibody (ANCA)-associated vasculitis; MPA: microscopic polyangiitis; GPA: granulomatosis with polyangiitis (Wegener's); EGPA: eosinophilic granulomatosis with polyangiitis (Churg-Strauss); ICV: Immune Complex Vasculitis; SVV: small vessel vasculitis; AGBM: anti-glomerular basement membrane disease; CV: Cryoglobulinemic Vasculitis; IGAV: IgA vasculitis (Henoch-Schonlein); HUV: Hypocomplementemic Urticarial Vasculitis; VVV: Variable Vessel Vasculitis; BD: Behcet's Disease; CS: Cogan's Syndrome; SOV: Single-Organ Vasculitis; CLA: Cutaneous Leukocytoclastic Angiitis; CA: Cutaneous Arteritis; PCNSV: Primary Central Nervous System Vasculitis; IA; Isolated Aortitis; CPPD: calcium-pyrophosphate-deposition; RS3PE: Remitting seronegative symmetrical synovitis with pitting edema; SAPHO: Synovitis-Acne-Pustulosis-Hyperostosis-Osteitis 
Inflammatory rheumatic diseases

Table 3. Comparative data of patients with inflammatory rheumatic diseases

\begin{tabular}{lccccc}
\hline Variable & $\begin{array}{c}\text { Germany } \\
\text { N= 25653 }\end{array}$ & $\begin{array}{c}\text { Netherland }^{\mathbf{1 8}} \\
\mathbf{N = 3 3 0 7 6}\end{array}$ & $\begin{array}{c}\text { Iran } \\
\text { N= 12626 }\end{array}$ & $\begin{array}{c}\text { Belgium }^{\mathbf{1 9}} \\
\text { N=1566 }\end{array}$ & $\begin{array}{c}\text { Nigeria }^{20} \\
\text { N=82 }\end{array}$ \\
\hline Female \% & 71.00 & - & 64.20 & - & - \\
Mean age at onset (years) & 51.50 & - & 41.17 & - & - \\
Rheumatoid arthritis\% & 50.6 & 45.2 & 47.30 & 45.8 & 25.6 \\
Systemic lupus erythamtosus \% & 4.72 & 1.7 & 8.10 & 1.9 & 12.1 \\
Systemic sclerosis \% & 1.2 & 0.6 & 2.36 & - & 7.3 \\
Spondyloarthropathies (total) \% & 22.61 & 20.8 & 17.23 & - & 4.8 \\
Ankylosing spondylitis \% & 5.79 & 8.6 & 5.26 & 10.2 & - \\
Reactive arthritis \% & 0.2 & 2.5 & 0.89 & 2.6 & 2.4 \\
Psoriatic arthritis \% & 8.26 & 6.1 & 2.95 & 8.2 & 2.4 \\
Sjogren's syndrome \% & 1.7 & 2.2 & 0.87 & 1.5 & 7.3 \\
Vasculitides \% & 2.1 & 1.3 & 6.83 & - & - \\
Behcet's disease \% & - & - & 4.18 & - & - \\
Juvenile idiopathic arthritis \% & - & 1.3 & 2.82 & - & - \\
Dermatomyositis/ polymyositis \% & - & - & 1.32 & - & 1.2 \\
Gout \% & 8.5 & 1.3 & 7.78 & 3.5 & 39.0 \\
Polymyalgia rheumatica \% & 3.8 & 5,6 & 0.41 & 5.6 & 2.4 \\
Overlap syndromes \% & & & 0.6 & & \\
\hline
\end{tabular}

In Nigeria [20], a study was performed to examine the prevalence and distribution of rheumatic diseases in a tertiary hospital outpatient practice. The study consisted of a small number of patients restricted to a tertiary institution in southwestern Nigeria, which, however, cannot represent the true prevalence of each rheumatologic disorder in the general community of the studied region.

Overall, inflammatory rheumatic diseases are more common in females than in males [21, 22]. In the current study, the proportion of women was lower compared to the study carried out in Germany (64\% versus $71 \%$ ).

Inflammatory rheumatic diseases can occur at any age [23] (age range in the current study was 1-93 years). In this study, the mean age at disease onset was 41 years, which was lower than in the study carried out in Germany (51 years). The reason for this difference may lie in the fact that the population of Iran is younger than the population of Germany.

About half of the patients with inflammatory rheumatic diseases have RA. The frequency of RA in this study $(47.5 \%)$ was slightly lower than that in the German study (50.6), but it was higher than its level in studies performed in Belgium (45.8\%) and the Netherlands (45.2\%).

The percentage of SLE (the second most common disease) was higher in the present study $(8.1 \%)$ than in the three other above-mentioned studies.

This difference can also be explained by the lower age of the Iranian population, since SLE is a disease of young adults.
The frequency of spondyloarthropathies was slightly lower in this study than in studies in Germany and the Netherlands. The most common subtype of spondyloarthropathies in this study was undifferentiated spondyloarthropathy, whereas the prevalence of ankylosing spondylitis in this study corresponded to its level reported in a study performed in Germany, i.e. less frequent than in the other two studies. The prevalence of arthritis in patients with psoriasis is $9.1 \%$ in Iran [24]. The frequency of PsA in the current study was less than three other studies.

Systemic sclerosis was seen more frequently in this study $(2.36 \%)$ than in three other studies.

The prevalence of Behcet's disease (BD) in Iran was 68 per 100,000 inhabitants, which is the second highest prevalence after Turkey (80-370 per 100,000) worldwide [25]. The frequency of Behcet's disease among patients in the present study was $4.18 \%$. The three other studies did not report any patients with Behcet's disease due to the rarity of $\mathrm{BD}$ in these countries. Behcet's disease is classified as a vasculitis [26]. It is possible that, in the aforementioned studies, $\mathrm{BD}$ was considered a vasculitis, and, therefore, its rate was not reported separately.

PMR was much less frequent in Iran $(0.4 \%)$ in comparison with other studies (Germany, 3.8\%; the Netherlands and Belgium, 5.6\%). PMR is a disease of the elderly. Thus, its current low prevalence can be associated with the younger population of Iran.

The prevalence of gout in Iranian population is $0.13 \%$ [25]. The frequency of gout in the present study matched its rate in the study carried out in Germany; 
however, it was higher than the other two studies.

Juvenile idiopathic arthritis (JIA) is the most common chronic pediatric rheumatologic disease. Only 359 cases of juvenile idiopathic arthritis were recorded here. The small number of such cases can be explained by the fact that the database is maintained in adult rheumatology units. During the last decade, the number of pediatric rheumatologists has increased in Iran; at present, most pediatric patients with rheumatologic problems are visited by pediatric rheumatologists.

Some patients who fulfill the criteria for a diagnosis of an autoimmune disease have overlapping features of a second autoimmune illness. In 30\%-52\% of patients with SLE, RA, or Sjögren's syndrome, the second autoimmune disease, rheumatic or nonrheumatic, will occur [27]. Sjogren's syndrome and APS can develop in many other IRDs. If SS or APS develops in patients with other IRDs, the diseases are called secondary Sjogren's syndrome or secondary APS. In this study, the association of these 2 disorders with any other IRDs as overlap syndromes was not taken into account. About $0.6 \%$ of patients in the current study had overlap syndromes. The most common overlap syndromes (2 IRDs) were RA-SLE (rhupus) and RA-SSc.

The database used in this study had several limitations: it only included patients visited by rheumatologists; it did not give information on the situation of patients who never reached the specialized sector. There is no information on how the diseases are diagnosed and treated at the level of the general population.

\section{Conflicts of interest}

The authors declare no conflicts of interest.

\section{Acknowledgments}

The authors are grateful to the patients for their cooperation.

\section{References}

1. Da Silva JAP, Woolf AD (eds.) Rheumatology in Practice, Springer, London; 2010.

2. Branco JC, Rodrigues AM, Gouveia N, et al. Prevalence of rheumatic and musculoskeletal diseases and their impact on health-related quality of life, physical function and mental health in Portugal: results from EpiReumaPt- a national health survey. RMD Open 2016; 2(1) e000166. doi: 10.1136/rmdopen2015-000166.

3. Fautrel B, Guillemin F. Cost of illness studies in rheumatic diseases. Curr Opin Rheumatol 2002; 14 (2): 121-6. doi: 10.1097/ 00002281-200203000-00008.

4. Glazier R, Fry J, Badley E. Arthritis and rheumatism are neglected health priorities: a bibliometric study. J Rheumatol 2001; 28 (4): 706-11.

5. Schumacher HR. Classification of rheumatic diseases. In: Clipped JH, Dieppe PA, (eds.) Rheumatology. Mosby, London; 1994: 7. 1-4.

6. Schirmer M. Advances in the Evaluation and Classification of Chronic Inflammatory Rheumatic Diseases. Discov Med 2012; 13(71): 299-304.

7. Andersson Gare B. Juvenile arthritis: who gets it, where and when? A review of current data on incidence and prevalence. Clin Exp Rheumatol 1999; 17: 36774.

8. Deane KD, Gabalawy HE. Pathogenesis and prevention of rheumatic disease: focus on preclinical RA and SLE. Nature Reviews Rheumatology 2014; 10 : 212-228. doi: 10.1038/nrrheum. 2014.6.

9. Raza K, Gerlag DM. Preclinical inflammatory rheumatic diseases. Rheumatic Diseases Clinics 2014; 40(4): 569-580. doi: http://dx.doi.org/10.1016/j.rdc.20 14.07.001.

10. Goldblatt F, O'Neill SG. Clinical aspects of autoimmune rheumatic diseases. The Lancet 2013; 382(9894): 797-808. doi: 10. 1016/S0140-6736(13)61499-3.

11. Jokar M, Mirfeizi Z. Epidemiology of vasculitides in Khorasan Province, Iran. Iranian Journal of Medical Sciences 2015; 40(4): 362-366.

12. Jokar MH, Mirfeizi Z. The demographic, clinical and laboratory profile of patients with giant cell arteritis: Case series. Sch. J. App. Med. Sci 2014; 2(5F): 1892-1895.

13. Jokar MH. Takayasu arteritis in Iran. SAS Journal of Medicine 2016; 2(3): 64-68.

14. Rezaiyazdi Z, Jokar MH, Saghafi M, Sahebary M, Nabavi S, Salari
M, Mirfeizi Z. Clinical and epidemiological manifestation of 100 patients with sarcoidosis and sarcoid arthritis. Medical Journal of Mashhad University of Medical Sciences 2015; 58 (5): 270-275.

15. Ermann J, Fathman CG. Autoimmune diseases: genes, bugs and failed regulation. Nat Immunol 2001; 2: 759-761. doi: 10.1038/ni0901-759.

16. Sheppeard H. The first 500 patients seen at a rheumatology clinic in a public hospital. $N \boldsymbol{Z}$ Med J 1986; 99: 716-18.

17. Zink A, Listing J, Klindworth C, Zeidler $\mathrm{H}$. The national database of the German Collaborative Arthritis Centres: I. Structure, aims and patients. Ann Rheum Dis 2001; 60(3): 199-206. doi: 10.1136/ard.60.3.199.

18. Miedema HS, van der Linden SM, Rasker JJ, Valkenburg HA. National database of patients visiting rheumatologists in the Netherlands: the standard diagnosis register of rheumatic diseases. A report and preliminary analysis. Br J Rheumatol 1998; 37(5): 555-61. doi: 10.1093/rheumatology/37.5.555.

19. Vanhoof J, Declerck K, Geusens P. Prevalence of rheumatic diseases in a rheumatologic outpatient practice. Ann Rheum 
Dis 2002; 61: 453-455. doi: 10 . 1136/ard.61.5.453.

20. Oguntona SA, Edunjobi AS, Olatunde AO. Prevalence of rheumatic diseases in a rheumatology outpatient practice of a tertiary hospital. International Research Journal of Medicine and Biomedical Sciences 2016; 1(2): 11-18.

21. Oliver JE, Silman AJ. Why are women predisposed to autoimmune rheumatic diseases? Arthritis Research \& Therapy 2009; 11(5):252. doi: 10.1186/ ar2825.

22. Vollenhoven RF. Sex differences in rheumatoid arthritis: more than meets the eye... BMC Medicine 2009; 7: 12. doi: 10.1186/1741-
7015-7-12.

23. See LC, Kuo CF, Chou MJ, Yu KH. Sex- and age-specific incidence of autoimmune rheumatic diseases in the Chinese population: a Taiwan populationbased study. Semin Arthritis Rheum 2013; 43: 381-6. doi: 10.1016/j.semarthrit.2013.06.001.

24. Jamshidi F1, Bouzari N, Seirafi $\mathrm{H}$, Farnaghi F, Firooz A. The prevalence of psoriatic arthritis in psoriatic patients in Tehran, Iran. Arch Iran Med 2008; 11(2): 1625. doi: 08112/AIM.008.

25. Davatchi F, Jamshidi AR, Banihashemi AT, Gholami J, Forouzanfar MH, Akhlaghi M. et al. Prevalence of Behcet's disease in Iran: a WHO-ILAR
COPCORD stage I study. APLAR Journal of Rheumatology 2007; 10:239243. doi: 10.1111/j.1479-8077. 2007.00295.x.

26. Jennette JC, Falk RJ, Bacon PA, Basu N, Cid MC, Ferrario F, et al. 2012 revised International Chapel Hill Consensus Conference Nomenclature of Vasculitides. Arthritis Rheum. 2013; 65:1-11. doi: 10.1002/art.37715.

27. Lockshin MD, Levine AB, Erkan D. Patients with overlap autoimmune disease differ from those with 'pure' disease. Lupus Science \& Medicine 2015; 2:e000084. doi: 10.1136/lupus2015-000084. 